Cartagena, 7 a 9 de septiembre de 2011

\title{
Sincronización de los ciclos de información y de gestión de infraestructuras de servicios hoteleros
}

\section{Synchronization of Information and Infrastructure Management Lifecycles in Hotel Services}

\author{
Alfonso Duran, Rajesh Natarajan y Esmeralda Giraldo \\ Área de Ingeniería de Organización, Escuela Politécnica Superior, Universidad Carlos III de Madrid. \\ Avda. de la Universidad, 30 289। I Leganés, Madrid. \\ duran@ing.uc3m.es
}

Fecha de recepción: 7-9-2011

Fecha de aceptación: 19-9-2011

Resumen: En muchas organizaciones de servicios, incluyendo las hoteleras, hay un desajuste temporal entre el ciclo de generación de información sobre sus clientes y el ciclo de decisiones de gestión de infraestructuras. Aquí abordaremos la sincronización de estos ciclos, mediante la utilización eficaz de sistemas de información. Analizaremos la problemática que dificulta hacer un uso más efectivo de estos datos. Seguidamente, apoyándonos en el ciclo de vida de los clientes, propondremos una clasificación de los datos sobre clientes vinculados a decisiones del hotel, así como de las decisiones para las que se usan. Asimismo, analizaremos cómo priorizar las actividades de gestión de datos para maximizar su contribución a la calidad de las decisiones.

Palabras clave: Sector hotelero, Gestión de datos, Infraestructura de servicios, Ciclo de vida de clientes, Ciclo de decisión, Sincronización de ciclos de vida.

\begin{abstract}
Most services operations, including those in the hospitality industry, suffer from a mismatch between the data/information generation and the infrastructure management decision cycles. Here, we address the synchronization of information and decision-making cycles through effective utilization of information systems. We discuss the issues hindering a more effective use of customer data. Then, using the customer lifecycle as the foundation, we provide a classification of hotel decision-related customer data and the supported infrastructure management hotel decisions. We also discuss how to prioritize the data management efforts to maximize their contribution to the decision making quality.
\end{abstract}

Key words: Hospitality Industry, Data Management, Service Infrastructure, Customer Life Cycle, Decision Cycle, Cycle Synchronization.

\section{Introducción}

Numerosas organizaciones del sector servicios operan en entornos caracterizados por ciclos de interacción con sus clientes dinámicos y continuados en el tiempo, y este entorno condiciona la forma en que toman sus decisiones sobre la gestión de sus infraestructuras. La mejora de sus procesos de decisión para explotar eficazmente la información existente exige que la información requerida esté accesible en el momento de la decisión. Ahora bien, una característica frecuente en estos entornos es un desajuste entre los ciclos de decisión y de disponibilidad de información. En muchos casos, la informa- ción requerida para apoyar una decisión solamente está disponible cuando ya ha pasado la ventana de decisión. Esto lleva a que las decisiones se tomen con menos información, disminuya su calidad y se pierdan oportunidades de generar ingresos mediante la venta de servicios adicionales. Considerando la intensa competitividad y el nivel de exigencia de los clientes en muchos sectores, como el hotelero, las empresas difícilmente pueden permitirse perder estas oportunidades.

En este trabajo, estudiaremos el desajuste entre los ciclos de información y de decisión y abordaremos su sincronización mediante la utilización eficaz de sis- 
temas de información. Utilizaremos el sector hotelero como ejemplo a lo largo de todo el trabajo; el sector hotelero resulta adecuado como ejemplo ilustrativo, ya que posee las características indicadas: ciclos de interacción con los clientes dinámicos y continuados en el tiempo, que podrían ser apoyados mediante una infraestructura de soporte a la decisión basada en datos. Para sobrevivir, los hoteles necesitan ser proactivos y flexibles. Este enfoque puede ayudarles a anticipar y personalizar sus productos y servicios en función de los requerimientos de sus clientes (Minghetti, 2003; Luck y Stephenson, 2009). Tras un análisis general del sector hotelero, discutiremos distintos aspectos de la problemática que deben abordar las empresas del sector hotelero, y cómo puede ayudarles la utilización más eficaz de los datos recogidos en el curso de sus operaciones.

Cualquier infraestructura de soporte a las decisiones para el sector hotelero debe estar focalizada en el cliente. Así pues, apoyándonos en el ciclo de vida del cliente, analizaremos en primer lugar las necesidades de decisión y la disponibilidad de información en las distintas etapas del ciclo de vida del cliente. Los dos ciclos considerados, el de datos sobre los clientes y el de decisiones que debe tomar el hotel, son considerados en paralelo, ya que el primero alimenta al segundo con información relevante para la toma de decisiones. Esto nos lleva a una clasificación de los procesos de toma de decisiones por parte del hotel. Esta clasificación se basa en la disponibilidad y suficiencia de datos sobre los clientes para la toma de decisiones en las distintas etapas del ciclo de vida de los clientes. Seguidamente analizaremos cómo los sistemas de información y tecnologías relacionadas pueden ayudar a cubrir las necesidades causadas por el desajuste entre los ciclos de información y de decisión.

El sector hotelero utiliza de forma generalizada sistemas de información (Sigala, Lockwood y Jones, 2001 ), tales como sistemas centralizados de reservas o sistemas integrados de «gestión de propiedades» (Property Management Systems, PMS). En el apartado correspondiente, realizaremos una breve revisión de los distintos sistemas de información utilizados en el sector hotelero, destacando los aspectos vinculados a la recolección de datos sobre clientes orientados a dar soporte a las decisiones. En el curso de sus operaciones diarias, estos sistemas capturan grandes cantidades de datos sobre los clientes. Los hoteles necesitan sacar partido a este recurso, asegurándose de que sus productos y servicios se ajustan a los requerimientos específicos de sus clien- tes. Ahora bien, para asegurar que los datos sobre clientes son usados de la forma más eficiente y eficaz, el proceso de gestión de datos debe alinearse con el de decisión. Esto es debido a que el valor potencial de los datos capturados sólo se materializa cuando se utilizan para apoyar la toma de decisiones. En este trabajo, analizaremos cómo las empresas sometidas a ciclos dinámicos de clientes pueden priorizar sus actividades de gestión de datos para facilitar que la toma de decisiones esté apoyada en datos reales, así como la problemática más relevante que implica dicha priorización.

\section{El sector hotelero}

En muchos países el sector turístico tiene una gran importancia y un peso significativo dentro de la economía (Rutherford y O'Fallon, 2007). En este trabajo nos referiremos al sector hotelero en una acepción amplia, incluyendo restauración y alojamiento. En los últimos años, el sector hotelero se ha expandido de forma no planificada, debido principalmente a las bajas barreras de entrada, originando un aumento del nivel de competencia. Los hoteles se diferencian entre sí en su tamaño (número de habitaciones) y en los servicios ofrecidos, tanto en la habitación en sí como en servicios auxiliares tales como restaurantes, centros de convención, posibilidades de juego, etc.

Algunos de los retos que debe afrontar el sector hotelero son típicos en el sector servicios. Una característica básica del hotel medio es que, al menos en el corto plazo, opera con una capacidad fija. Cualquier incremento de su capacidad requiere una expansión de su infraestructura física (edificios, infraestructura de servicios, ...), que caso de ser abordada se hace en bloques (no de forma continua) e involucra importantes inversiones. Por tanto, la expansión de la capacidad sólo se decide tras un cuidadoso análisis de los patrones de demanda actual y proyectada. Por otra parte, una pernoctación es un bien «perecedero»; una pernoctación que no se vende se pierde, ya que no puede ser almacenada. Esto contribuye a una mayor competencia entre los hoteles para maximizar los niveles de ocupación.

El sector hotelero tiene una demanda fluctuante, que varía con la estación, el día del mes y de la semana. Asimismo, la demanda de cada segmento de clientes (viajeros de negocios, de ocio, etc.) es diferente de la de los demás segmentos, en aspectos como tarifas, antelación de las reservas, servicios requeridos, etc. Las habitaciones típicamente se venden con an- 
telación, ya que el hotel debe encontrar un equilibrio entre la incertidumbre sobre el nivel de ocupación y las tarifas que puede conseguir. Dada la heterogeneidad de la demanda, la previsión de la demanda del hotel es compleja. Por otra parte, el hotel vende a sus clientes también otros servicios, como restaurante, lavandería, salas de convenciones, paquetes turísticos etc. Eso introduce otro elemento en el equilibrio a alcanzar, ya que la posibilidad de que un cliente utilice esos servicios incrementa el interés en atraerle como huésped. Una fuente adicional de presión a la baja sobre las tarifas hoteleras es la creciente transparencia, en términos de tarifas y de disponibilidad, causada por Internet y los intermediarios de información que operan en ella. Costes de transacción muy bajos permiten a los clientes reservar una habitación, buscar una oferta mejor, cancelar y re-reservar. Estos factores complican el problema de maximización de los ingresos/beneficios del hotel, exigiendo abordar de forma proactiva y flexible la anticipación de los requerimientos de los clientes y la correspondiente adaptación de los productos y servicios del hotel.

\section{Captura de datos y decisiones por parte del hotel}

Los hoteles acceden a una gran cantidad de datos sobre sus clientes, capturados por los diversos sistemas de información que usan como apoyo a sus operaciones. Cada uno de esos sistemas, tales como sistemas centralizados de reservas, sistemas «de gestión de propiedades» (Property Management Systems, PMS), sistemas de «Yield Management» y otros, se centra en un aspecto diferente de las operaciones del hotel, y por tanto tiene una visión parcial del cliente. En consecuencia, para obtener una visión integrada del cliente, nos apoyaremos en el ciclo de vida del cliente para la definición y el análisis de los requerimientos de información sobre los clientes para la toma de decisiones.

El ciclo de vida del cliente muestra las varias etapas que atraviesa un cliente cuando es atendido por un hotel. Cada etapa, caracterizada por múltiples interacciones posibles con el hotel y su personal, incluye oportunidades de influir en el huésped a través de decisiones relacionadas con el cliente. La primera etapa es el proceso de «búsqueda», centrado fundamentalmente en información sobre el hotel, como disponibilidad, tarifas y servicios complementarios, que permiten a un cliente evaluar la oferta de un hotel. Esta búsqueda puede realizarse con ayuda de una agencia de viajes, o mediante una búsqueda en Internet; numerosos hoteles y servicios web ofrecen menús de búsqueda que facilitan este proceso. Si las necesidades del cliente son cubiertas con los resultados de esta búsqueda, puede contactar directamente al hotel. Si el hotel dispone de suficiente información sobre las necesidades y prioridades del cliente, puede incrementar las probabilidades de que efectúe la reserva, adecuando su oferta a sus intereses específicos. La siguiente etapa de interacción se corresponde con la llegada y registro en el hotel. Además de asignarle la habitación adecuada, el hotel puede proporcionar información adicional relativa a sus servicios auxiliares, así como eventuales ofertas para estos. Durante el resto de la estancia, el huésped puede asimismo utilizar los diversos servicios del hotel; durante esta etapa hay numerosas ocasiones para el hotel, tanto para ofrecer sus servicios como para recolectar información sobre el cliente y sus preferencias, que pueda posteriormente utilizar para afinar mejor sus decisiones. El proceso de registro de salida es la última oportunidad de interacción con el cliente en una estancia. La información de realimentación conseguida del cliente en esta eta$\mathrm{pa}$, o con posterioridad, puede ayudar al hotel a refinar el ajuste de sus servicios a los gustos y preferencias de los clientes. Las opiniones de los clientes en los distintos foros de opinión existentes en Internet pueden influir en las decisiones de los nuevos clientes durante su proceso de búsqueda. Por otra parte, en ocasiones, el cliente que ya ha realizado un reserva puede cancelarla e iniciar un nuevo proceso de búsqueda en un momento posterior. En la Figura I, las etapas del ciclo de vida se muestran dentro del ciclo de vida de los clientes actuales, y están referenciadas mediante números.

Dentro del ciclo de vida del cliente, podemos considerar la recogida de datos sobre el huésped y la toma de decisiones de gestión de infraestructuras vinculadas al huésped por parte del hotel como dos ciclos paralelos. El ciclo de captura de datos representa los distintos momentos en los que datos/ información sobre el cliente son recogidos por el hotel. Análogamente, el ciclo de decisión incluye los momentos en los que el hotel toma decisiones de gestión relacionadas con el cliente. La calidad de dichas decisiones puede mejorar sustancialmente si, en el momento de tomarlas, el decisor tiene acceso a los datos de apoyo relevantes. Esto requeriría que el ciclo de captura de datos estuviera sincronizado con el ciclo de decisión. La Figura I muestra gráficamente las actividades de recogida de datos y de decisión del hotel en el marco del ciclo de vida del cliente. Asu- 
Figura I

El proceso de recolección de datos y toma de decisiones desde la perspectiva del ciclo de vida del cliente

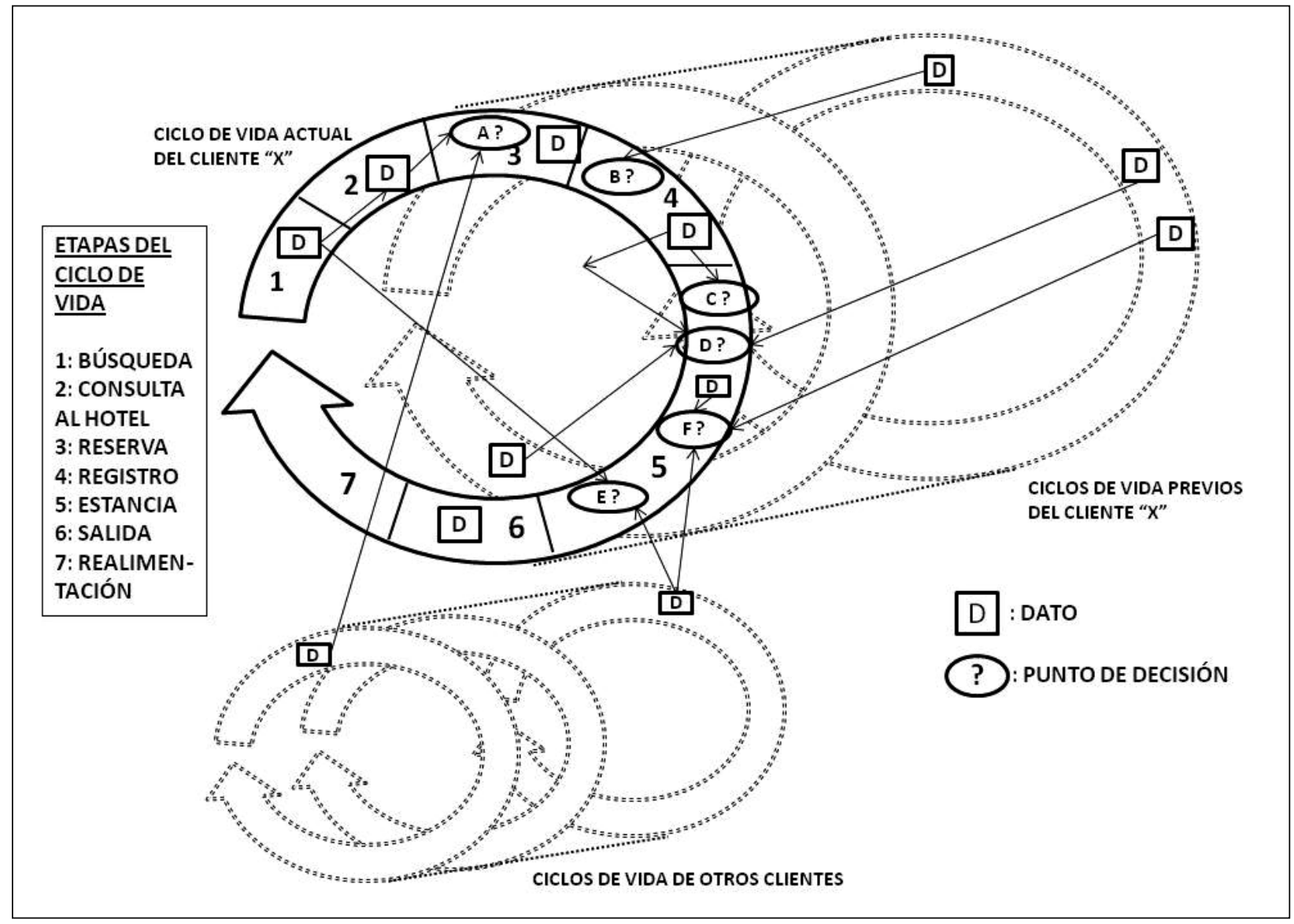

mamos que $X$ es un cliente alojado en el hotel, sobre el cual el hotel debe tomar una decisión. El hotel ha podido recoger información sobre el cliente $X$ durante su ciclo actual, por ejemplo a medida que ha utilizado, o no, las distintas infraestructuras de servicios del hotel. Adicionalmente, puede conservar datos sobre este mismo cliente, procedentes de eventuales estancias anteriores en el hotel. Estos ciclos de vida previos del cliente $X$ están representados en la Figura I en trazos discontinuos. Así, para un cliente $X$, podemos distinguir entre los datos del ciclo de vida actual (DCA) y los datos de ciclos previos (DCP). El hotel también recoge datos operativos y de comportamiento sobre el resto de los clientes durante sus respectivas estancias/ ciclos de vida. Estos datos podrían denominarse datos sobre otros clientes (DOC). En la Figura I se analiza la dimensión temporal del proceso de decisión del hotel respecto al de captura de datos y la disponibilidad de información relevante durante el ciclo de vida de un cliente tipo. Los datos recolectados por el hotel se representan mediante rectángulos, y los puntos de decisión mediante óvalos.

\section{Tipología de decisiones en función de los datos de apoyo}

La calidad de la toma de decisiones mejora si los datos relevantes sobre los clientes están disponibles en el momento de la decisión. En el curso normal de sus operaciones, el hotel puede recolectar datos tales como nombre, edad o dirección directamente de sus huéspedes durante el ciclo/ estancia actual. Algunas decisiones pueden apoyarse directamente en estos datos del ciclo de vida actual (DCA). Así, el servicio de habitaciones puede preparar un desayuno o repartir un periódico ajustado al gusto de un cliente concreto preguntándole directamente por su preferencia. En la Figura I, el punto de decisión C representa una situación de este tipo, a las que denominaremos decisiones de Categoría l. En ocasiones, el hotel puede basar sus decisiones en datos recogidos durante visitas previas de ese mismo cliente, esto es, datos de ciclos previos (DCP) del cliente $X$. Considérese, como ilustración, un punto de decisión $B$ durante la etapa de registro del ciclo de cliente actual. Por ejemplo, el hotel está preparando la habitación para 
la estancia de un cliente y quiere ajustarla a las preferencias y gustos individuales de ese cliente. Para ello, el hotel puede utilizar información recogida en visitas previas del cliente, tales como preferencias de almohada o elección de balcón, para seleccionar y preparar la habitación para esta visita. Estas decisiones están basadas en datos de ciclos previos (DCP) del cliente $X$, y nos referiremos a ellas como decisiones de Categoría II. En este caso, el hotel usará DCP ya que aún no ha tenido ocasión de recoger los datos relevantes para esta decisión en el ciclo actual (DCA).

Supóngase ahora que el hotel quiere estimar cuántos suministros va a consumir el cliente $X$ durante la visita actual. El dato de consumo real sólo estará disponible al final de la estancia. No disponiendo aún de ese dato, el hotel puede extrapolar el consumo probable a partir de los datos de visitas previas (DCP) y del uso actual de los primeros días de la estancia actual (DCA). Estas decisiones, que requieren tanto DCP como DCA, son también de Categoría II, dado que asumiremos que todos los datos ya recogidos en el ciclo actual (DCA) están disponibles en cualquier punto para apoyar la toma de decisiones. Esta situación está representada, en la Figura I, por el punto de decisión D.

Consideremos ahora una variable como la Disposición a Gastar en otros servicios del hotel, tales como restaurante, Spa, etc. Para un cliente nuevo, el hotel sólo sabría si tiene alta o baja disposición una vez que haya utilizado, o no, dichos servicios. Ahora bien, usando técnicas de clasificación y agrupación (clustering), el hotel puede predecir, con un cierto grado de precisión, el comportamiento del cliente a partir del comportamiento de otros clientes similares. Para identificar otros clientes que son similares al cliente actual $X$, esto es, aquellos clientes cuyos datos deberían usarse como base para la extrapolación, el hotel necesita tener información sobre ciertas características del cliente $X$, esto es, DCA. Estas decisiones, que requieren tanto DCA como datos sobre otros clientes (DOC) pueden denominarse decisiones de Categoría III, y estarían representadas por el punto de decisión E en la Figura I.

Muchos hoteles ofrecen a sus clientes descuentos en sus servicios auxiliares, como restauración, gimnasio, spa, casino etc. Dado que el personal del hotel normalmente no dispone de suficiente tiempo para describir y destacar todos los servicios del hotel, puede centrarse en promocionar y ofrecer descuentos en sólo algunos de sus servicios, que deberían ser aquellos que con mayor probabilidad sean de interés para el cliente durante su estancia actual. Esto es, el hotel desearía conocer la propensión a gastar del cliente en cada uno de sus servicios antes de que éste los haya usado. Dado que el dato real no está aún disponible, el hotel podría extrapolar la probabilidad de que el cliente use cada servicio concreto a partir de los servicios que dicho cliente hubiera utilizado en sus visitas anteriores (DCP) y de información sobre otros clientes «similares» (DOC). Estas decisiones, que requieren tanto DCP como DOC, pueden denominarse decisiones de Categoría IV, representadas por el punto de decisión F en la Figura I. Frecuentemente, este tipo de decisiones también utilizan DCA para derivar los datos en los que apoyarán sus decisiones. La Tabla I resumen las distintas categorías de decisiones.

Tabla I

Clasificación de decisiones del hotel vinculadas a clientes

\begin{tabular}{|l|l|l|l|}
\hline \multicolumn{1}{|c|}{$\begin{array}{c}\text { CATEGORÍA } \\
\text { DE DECISIÓN }\end{array}$} & \multicolumn{1}{|c|}{ DATOS DE APOYO } & $\begin{array}{c}\text { MECANISMOS DE DERIVACIÓN } \\
\text { DE LOS DATOS }\end{array}$ & \multicolumn{1}{|c|}{ EJEMPLO } \\
\hline Categoría I & DCA & Conocimiento del cliente & • Preferencia en bebidas \\
\hline Categoría II & DCP (DCA + DCP) & $\begin{array}{l}\text { Extrapolación directa del comporta- } \\
\text { miento pasado, conocimiento del } \\
\text { cliente. }\end{array}$ & $\begin{array}{l}\text { P Preferencias sobre disposición de la } \\
\text { habitación, como tipo de almohada. } \\
\text { Estimación del consumo de sumi- } \\
\text { nistros durante la visita actual. }\end{array}$ \\
\hline Categoría III & DCA + DOC & $\begin{array}{l}\text { Conocimiento de relaciones, extrapo- } \\
\text { lación y métodos de inferencia prove- } \\
\text { nientes de la minería de datos, la es- } \\
\text { tadística y campos relacionados. }\end{array}$ & $\begin{array}{l}\text { Clasificación de un cliente en función } \\
\text { de su «Disposición a gastar». }\end{array}$ \\
\hline Categoría IV & $\begin{array}{l}\text { DCP + DOC } \\
\text { (DCA + DCP + DOC) }\end{array}$ & $\begin{array}{l}\text { Conocimiento de relaciones, extrapo- } \\
\text { lación y métodos de inferencia prove- } \\
\text { nientes de la minería de datos, la es- } \\
\text { tadística y campos relacionados }\end{array}$ & $\begin{array}{l}\text { Selección de las «ofertas» a realizar } \\
\text { a un cliente }\end{array}$ \\
\hline
\end{tabular}

DCA: Datos del Ciclo Actual del cliente «X»

DCP: Datos de Ciclos Previos del cliente «X»

DOC: Datos sobre Otros Clientes 
Los Datos del Ciclo de Vida Actual, DCA, son la base preferible para apoyar la toma de decisiones, ya que son los datos más actualizados pertenecientes directamente al cliente respecto al cual se toma la decisión. Ahora bien, como se ha comentado, frecuentemente estos datos sólo están disponibles en un momento del ciclo de vida del cliente posterior a la decisión. Cabe destacar que esta situación no es atribuible a un mal funcionamiento de los mecanismos de captura de datos del hotel, sino que responde a una limitación intrínseca derivada del desajuste entre el ciclo de decisión y el de captura de datos. Para salvarla, las empresas apoyan sus decisiones en la extrapolación e inferencia a partir de DCP y DOC que fueron capturados en su momento por los procedimientos de registro de datos de la empresa. La minería de datos, la estadística y otras técnicas predictivas pueden aplicarse para extrapolar datos para el soporte de las decisiones a partir de DCP y DOC (Magnini, Honeycutt y Hodge, 2003; Law, Henry y Carey, 2007).

\section{Sistemas de Información en el sector hotelero}

La gestión efectiva de las infraestructuras de servicios de un hotel exige un flujo sincronizado de datos sobre sus clientes entre sus distintos departamentos. La información relativa al servicio proporcionado a un cliente por un departamento será utilizada por el siguiente departamento en la cadena de servicios. Por ejemplo, la información sobre reservas permite al departamento de limpieza de habitaciones tener las habitaciones listas para los clientes en el momento de su llegada y registro. Para apoyar sus operaciones, los hoteles utilizan diversos sistemas de información, tales como sistemas centralizados de reservas, sistemas de «gestión de propiedades» (Property Management Systems, PMS), sistemas de «Yield/ Revenue Management», sistemas de gestión de las relaciones con los clientes (Customer Relationship Management, CRM), sistemas integrados de gestión (Enterprise Resource Planning, ERP), etc. (Buhalis y Law, 2008; Law y Jogaratnam, 2005). También utilizan sistemas más especializados, como sistemas de entretenimiento (Guest Entertainment Systems), sistemas de seguridad y control de accesos, etc. La utilización de estos sistemas puede incrementar la eficiencia, a través de un control más estrecho de las operaciones conseguido mediante mecanismos de coordinación más eficaces y de menor coste (Law y Jogaratnam, 2005).

Los sistemas centralizados de reservas (Central Reservation Systems, CRS) almacenan y distribuyen in- formación sobre un hotel, tal como información sobre reservas, inventario de habitaciones, perfiles de los huéspedes, etc. Estos sistemas ayudan a reducir el coste del proceso de reservas, facilitan el proceso de referencia de clientes entre hoteles de la misma cadena y facilitan el seguimiento de las campañas de marketing. Asimismo distribuyen la misma información a través de múltiples canales, tales como agentes, página web del hotel, centros de reservas (Call Centers), etc., por lo que dan a los clientes una imagen única y consistente del hotel y sus características. La gestión de la demanda es particularmente importante en el sector hotelero dado que, en el corto plazo, el nivel de oferta está prefijado. Los sistemas de gestión de ingresos (Revenue Management / Yield Management, RM/YM) maximizan la ocupación de las habitaciones y por tanto los ingresos generados por la infraestructura fija del hotel, ajustando el diseño de las tarifas a las características cambiantes de la demanda (Noone, Kimes y Renahan, 2003). Los sistemas «de gestión de propiedades» (PMS) facilitan la gestión de los activos de un hotel, incluyendo el mantenimiento, cumplimiento de normativa, gestión del personal, etc. Estos sistemas dan soporte a múltiples actividades, incluyendo las reservas de los clientes, las reservas vía web, la gestión de los sistemas de punto de venta, etc. Los PMS pueden interactuar con otros sistemas, como sistemas centralizados de reservas y sistemas YM/RM, colaborando así a mantener la consistencia de tarifas e información sobre disponibilidad en toda la cadena.

El desarrollo de Internet y de los canales de distribución asociados ha cambiado la forma en la que los clientes buscan la información sobre los hoteles, comparan tarifas y reservan habitaciones. La aparición de intermediarios que combinan información proveniente de distintas cadenas hoteleras y ofrecen comparativas de precios, así como agresivos descuentos, ha introducido restricciones adicionales en las tarifas hoteleras ( $O^{\prime}$ Connor y Murphy, 2004). Los hoteles necesitan gestionar su presencia en la red manteniendo actualizada la información sobre sus servicios en sus páginas web y ofrecer a los distintos clientes la posibilidad de realizar diversas transacciones en línea. Al mismo tiempo, deben diseñar sus ofertas para intentar evitar perder clientes a manos de intermediarios virtuales y gestionar el efecto a la baja que tienen sobre sus tarifas (Carroll y Siguaw, 2003).

Los sistemas de gestión de las relaciones con los clientes (CRM) apoyan a los hoteles en el proceso de transformar a los potenciales huéspedes en clientes leales y rentables, gestionando los múltiples aspectos de su relación (Minghetti, 2003; Luck y Stephenson, 
2009). Los sistemas CRM ayudan a presentar una imagen consistente, al permitir al personal del hotel en contacto con un cliente acceso a toda la información generada durante las interacciones previas con dicho cliente. Con la ayuda de dicha información, el personal del hotel puede adaptar sus reacciones para ajustarse a los gustos y preferencias revelados por el cliente. Los elevados volúmenes de datos sobre clientes recogidos por los sistemas CRM y el resto de los sistemas contienen abundante información, potencialmente valiosa. Diversas empresas han invertido en sistemas de Inteligencia de Negocio y sistemas relacionados como Almacenes de Datos, Minería de Datos, etc., que analizan los datos sobre los clientes en un intento de predecir su comportamiento (Magnini, Honeycutt y Hodge, 2003; Law, Henry y Carey, 2007). Si esta información sobre los clientes es realimentada al resto de los sistemas, el personal del hotel puede ajustar consecuentemente su actuación, incrementando así su adecuación a las características específicas de cada cliente.

\section{Sincronización de la captura de datos como apoyo de la toma de decisiones}

La infraestructura de gestión de datos de los hoteles debe evaluarse en función de su contribución a la toma de decisiones, y no por el volumen de datos que capture o difunda. Las empresas hoteleras suelen tener diversos sistemas de información que apoyan sus operaciones diarias. Algunos sistemas actúan como fuentes de datos para otros; así, los sistemas de gestión de propiedades pueden alimentarse de datos procedentes de los sistemas de reservas. Consecuentemente, la eficaz integración de los distintos sistemas individuales es una precondición para explotar plenamente el potencial de soporte a la decisión de los datos de clientes.

El marco de referencia y la tipología de categorías de decisión discutidas en los apartados previos proporcionan un punto de partida útil para la priorización de los requerimientos de datos. El hotel debe identificar aquellos procesos de decisión que más se beneficiarían de la disponibilidad de datos de soporte. No todas las decisiones son iguales, en aspectos tales como su importancia, criticidad o el valor que potencialmente añaden al hotel. Así, las decisiones difieren en su alcance, duración del impacto, riesgo y beneficio. Algunas decisiones, de alcance estratégico, afectan a múltiples clientes y no son fácilmente reversibles, como la elección del mobiliario o la distribución de las habitaciones. Otras pueden proporcionar al hotel oportunidades de in- gresos adicionales, como la oferta de descuentos en servicios de interés para el cliente. En cambio, el hotel no obtendrá ganancias de promocionar entre sus clientes servicios que no se adecúen a sus intereses. Si se considera ahora la organización de los servicios de limpieza, dado que se da por supuesto que las habitaciones deben estar limpias, una decisión adecuada puede no tener impacto económico; ahora bien, reducir los servicios de limpieza por debajo de un cierto nivel puede llevar a clientes descontentos y afectar negativamente a los ingresos futuros. Por tanto, tanto los beneficios como los riesgos potenciales asociados a una decisión deben influir en su eventual inclusión en la lista de procesos de decisión importantes, que deben ser adecuadamente apoyados por la infraestructura de gestión de datos del hotel.

Seguidamente, el hotel debe evaluar sus mecanismos actuales de recogida y diseminación de datos, desde la perspectiva de su importancia y significatividad para los procesos más relevantes de decisión, dando prioridad a los datos que apoyan las decisiones más críticas. Asimismo, aquellos datos que dan soporte a múltiples decisiones tienen mayor valor desde una perspectiva decisional. Algunos tipos de datos necesitan ser actualizados a intervalos regulares para mantener su validez y su relevancia para las decisiones. Así pues, la decisión de recoger y mantener cada tipo de dato sobre los clientes debe estar basado en un análisis coste-beneficio sobre las diferentes decisiones soportadas por dicho dato. Por ejemplo, requerimientos concretos de los clientes pueden ser satisfechos por el hotel sin necesidad de información sobre sus preferencias; consecuentemente, sería innecesario recoger previamente información sobre estas preferencias.

Los Datos del Ciclo de Vida Actual, DCA, son los más relevantes desde el punto de vista del apoyo la toma de decisiones. Ahora bien, debido al desajuste entre los ciclos de decisión y de captura de datos, esos datos directos pueden no estar disponibles cuando se requieren. En esos casos, las decisiones se apoyan mediante la extrapolación a partir de datos de ciclos previos (DCP) y datos de otros clientes (DOC). La utilidad de dichos datos depende no sólo de cuán apropiado sea su proceso de generación, sino también de la exactitud y relevancia de los DCP y DOC utilizados para generarlos. Así, los datos provenientes de una estancia muy antigua pueden no ser muy fiables para predecir el comportamiento actual. Por tanto, para ser efectivos, los mecanismos de creación y extrapolación de los datos necesitan actualizaciones periódicas, y sus mecanismos de diseminación deben estar sincronizados con los ciclos de decisión del hotel. 


\section{Resumen}

El gran volumen de datos que el sector hotelero obtiene sobre sus clientes en el curso de sus operaciones diarias puede ser explotado para mejorar la toma de decisiones. Ahora bien, debido al desajuste temporal intrínseco entre los ciclos de decisión y de captura de datos, los datos relevantes pueden no estar disponibles cuando deben ser usados para una decisión. El uso eficaz de sistemas de información puede aliviar en cierta medida este problema. En este artículo se analiza en primer lugar los procesos de recogida de datos y de toma de decisiones de gestión de infraestructuras en un hotel tipo utilizando el ciclo de vida del cliente como base. Esto lleva a una clasificación de las decisiones en función de la información en que se apoyan. La falta de disponibilidad de datos del ciclo actual (DCA) puede ser parcialmente compensada extrapolando los datos requeridos a partir de datos de ciclos previos (DCP) y datos sobre otros clientes (DOC). Este proceso puede apoyarse en técnicas como minería de datos, técnicas estadísticas o inteligencia artificial.

Una estrecha integración de los distintos sistemas de información utilizados en el sector hotelero facilita el proceso de poner a disposición del decisor los datos relevantes durante la ventana temporal de decisión. Al igual que las diversas decisiones tienen diferente criticidad e importancia para el hotel, los diferentes elementos de datos pueden implicar costes muy diferentes para su captura, mantenimiento, derivación y diseminación. Dada la limitación de recursos, la mejora de las decisiones del hotel mediante el uso de datos de soporte requiere una priorización de las actividades de gestión de datos de acuerdo a la criticidad de las decisiones soportadas por dichos datos. Puede haber una mejora sustancial en la calidad de las decisiones si las decisiones más importantes son apoyadas mediante la disponibilidad de los datos relevantes. Una posible línea futura de investigación es el estudio de la cuantificación del grado de soporte a la decisión que proporcionan los distintos elementos de datos, y la consiguiente evaluación de la infraestructura de gestión de datos de un hotel desde la perspectiva de su capacidad para apoyar y mejorar la toma de decisiones.

\section{Agradecimientos}

Esta investigación ha sido financiada por el Plan $\mathrm{Na}$ cional de I+D+i, proyecto «Optimización de la asignación de infraestructuras de servicios mediante si- mulación —-sectores hotelero y sanitario.») (DPI200804872).

\section{Bibliografía}

BUHALIS, D., y LAW, R. (2008) «Progress in information technology and tourism management: 20 years on and 10 years after the Internet - The state of eTourism research». Tourism Management, 29, pp. 609-623.

CARROLL, B.y SIGUAW, J. (2003) «The evolution of electronic distribution: Effects on Hotels and Intermediaries». Cornell Hotel and Restaurant Administration Quarterly, August, 2003, pp. 38-50.

LAW, R., HENRY, M. y CAREY, G. (2007) «Data Mining in Tourism Demand Analysis: A Retrospective Analysis». In R. Alhajj et al. (Eds.) Springer, ADMA 2007, LNAI 4632, pp. 508-5I 5.

LUCK, D. y STEPHENSON, M.L. (2009) «An evaluation of the significant elements of customer relationship management within the hotel industry». Tourism Today. Fall 2009, pp. 7-26.

MAGNINI, V.P., HONEYCUTT Jr., E.D. y HODGE, S. K., (2003). «Data Mining for Hotel Firms: Use and Limitations.» Cornell Hotel and Restaurant Administration Quarterly, 44(2), pp. 99-105.

MINGHETTI, V. (2003) «Building Customer Value in the Hospitality Industry:Towards the definition of a Customer-centric Information System». Information Technology \& Tourism. 6, pp. |4|-152.

NOONE, M. B., KIMES E.S., y RENAHAN, L.M.I. (2003). «Integrating customer relationship management and revenue management: A hotel perspective». Journal of Revenue and Pricing Management, 2(I), pp. 7-21

RUTHERFORD, D.G. y O'FALLON, M.J. (ed.) (2007). Hotel Management and Operations. 4th edn. John Wiley and Sons, Inc. New Jersey.

SIGALA, M., LOCKWOOD A., y JONES, P. (200I) «Strategic implementation and IT: gaining competitive advantage from the hotel reservations process». International Journal of Contemporary Hospitality Management, |3(7), pp. 363-37|.

LAW, R., y JOGARATNAM, G. (2005) «A study of hotel information technology applications». International Journal of Contemporary Hospitality Management, 17(2), pp. 170-180.

O'CONNOR, P. y MURPHY, J. (2004) «Research on information technology in the hospitality industry». International Journal of Hospitality Management, 23, pp. 473-484. 